\title{
Formation and Function of Synapses with Respect to Schwann Cells at the End of Motor Nerve Terminal Branches on Mature Amphibian (Bufo marinus) Muscle
}

\author{
Greg T. Macleod, Paul A. Dickens, and Max R. Bennett \\ The Neurobiology Laboratory, Department of Physiology and Institute for Biomedical Research, University of Sydney, \\ NSW 2006 Australia
}

\begin{abstract}
A study has been made of the formation and regression of synapses with respect to Schwann cells at the ends of motor nerve terminal branches in mature toad (Bufo marinus) muscle. Synapse formation and regression, as inferred from the appearance and loss of $\mathrm{N}$-(3-triethylammoniumpropyl)-4-(4-(dibutylamino) styryl) pyridinium dibromide (FM1-43)-stained vesicle clusters, occurred at the ends of terminal branches over a $16 \mathrm{hr}$ period. Multiple microelectrodes placed in an array about FM1-43 blobs at the ends of terminal branches detected the electrical signs of neurotransmitter being released onto receptors. Injection of a calcium indicator (Oregon Green 488 BAPTA-1) into the motor nerve with subsequent imaging of the calcium transients, in response to stimulation, often showed a reduced calcium influx in the ends of terminal branches. Injection of a fluorescent dye into motor nerves revealed the full extent of their terminal branches
\end{abstract}

Changes in the patterns of synapses in the relatively mature peripheral nervous system may occur over periods of weeks or months in autonomic ganglia (Purves et al., 1987) and motor nerve terminals of mice (Lichtman et al., 1987; Robbins and Polak, 1988; Wigston, 1989; Balice-Gordon and Lichtman, 1990), as well as at amphibian motor nerve terminals (Wernig et al., 1980; Anzil et al., 1984). Such changes can be triggered by physical lesions to surrounding nerves or by perturbation of the system after insertion of neural implants (Ko and Chen, 1996) or sources of growth factor (Trachtenberg and Thompson, 1997). In the mammalian cornea, synaptic remodeling has been observed in sensory nerve endings over periods as short as a few hours (Harris and Purves, 1989), as have changes in the pattern of synapses on neurons in the hippocampus (Engert and Bonhoeffer, 1999; Maletic-Savatic et al., 1999; Toni et al., 1999). The question of whether synapse formation and regression occurs over periods of a few hours at mature motor nerve terminals has not been investigated in detail. We show that such remodeling is a constant feature of mature nerve terminals in vitro, with the formation and regression of terminal processes revealed through injection of fluorescent dyes. Calcium imaging techniques, as well as the application of styryl dyes and multi-electrode recording techniques, show the remodeling extremities to be functional.

The terminal Schwann cell (TSC) at the motor nerve terminal has been revealed as the substrate for nerve growth during

Received Aug. 25, 2000; revised Dec. 6, 2000; accepted Jan. 4, 2001.

Correspondence should be addressed to M. R. Bennett at the above address. E-mail: maxb@physiol.usyd.edu.au.

Copyright (C) 2001 Society for Neuroscience $0270-6474 / 01 / 212380-13 \$ 15.00 / 0$ and growing processes. Injection of the terminal Schwann cells (TSCs) often revealed pseudopodial TSC processes up to $10-\mu \mathrm{m}-$ long. Imaging of these TSC processes over minutes or hours showed that they were highly labile and capable of extending several micrometers in a few minutes. Injection of motor nerve terminals with a different dye to that injected into their TSCs revealed that terminal processes sometimes followed the TSC processes over a few hours. It is suggested that the ends of motor nerve terminals in vivo are in a constant state of remodeling through the formation and regression of processes, that TSC processes guide the remodeling, and that it can occur over a relatively short period of time.

Key words: synapses; Schwann cells; motor nerve; formation; regression; Bufo marinus reinnervation (Son and Thompson, 1995; O’Malley et al., 1999). Because glial cells have been shown to play an active role in neurotransmission at motor nerve terminals (Robitaille, 1998), it was of interest to see whether active remodeling of the TSC is accompanied by remodeling of the terminal processes and their synaptic contacts at intact motor nerve terminals. We have therefore injected single TSCs, as well as motor nerve terminals, with fluorescent dyes to determine the relationship between TSCs and the remodeling terminal processes.

\section{MATERIALS AND METHODS}

Preparation and solutions. Experiments were performed on the flexor brevis digitorum $\mathrm{V}$ muscle, the lumbricalis digiti $\mathrm{V}$ muscle, or the ilio-fibularis muscle (Ecker, 1889) of the cane toad Bufo marinus. They were collected from their natural environment in northeastern Australia and experimented on within 6 weeks of collection. The motor nerve terminal in these animals may change its properties with different seasons (Bennett et al., 1991). Accordingly, the seasons of collection are specified in Results. Animals were between 40 and $70 \mathrm{~mm}$ in length and killed by double-pithing. Muscles were dissected from the hind limb with up to 1 $\mathrm{cm}$ of nerve attached and pinned on a silicone elastomer (SYLGARD; Dow Corning, Midland, MI) bed in an organ bath. Ringer's solution containing (in mM): $\mathrm{NaCl} 111.2, \mathrm{KCl} 2.5, \mathrm{NaH}_{2} \mathrm{PO}_{4} 1.5, \mathrm{NaHCO}_{3} 16.3$, glucose 7.8, and $\mathrm{MgCl}_{2} 1.2$, bubbled with a gas mixture of $95 \% \mathrm{O}_{2}$ and $5 \% \mathrm{CO}_{2}$, constantly perfused the organ bath at a rate of $3 \mathrm{ml} / \mathrm{min}$. The temperature was maintained at $\sim 18^{\circ} \mathrm{C}$. The concentration of calcium ions in the Ringer's solution $\left(\left[\mathrm{Ca}^{2+}\right]_{\mathrm{o}}\right.$ ) was $0.4 \mathrm{~mm}$ for all electrophysiological experiments and $1.8 \mathrm{~mm}$ for all other experiments unless otherwise indicated.

Fluorescent probes. The hexapotassium salt form of the calciumsensitive dyes [Oregon Green 488 BAPTA-1 (OG-1) and Oregon Green 488 BAPTA-5N (OG-5N)] and the sodium salt form of the calciuminsensitive dyes [Alexa Fluor 488 hydrazide (AF488) and Alexa Fluor 568 hydrazide (AF568)] were purchased from Molecular Probes (Eu- 
gene, OR). $N$-(3-triethylammoniumpropyl)-4-(4-(dibutylamino)styryl) pyridinium dibromide (FM1-43) was also purchased from Molecular Probes. Motor nerve terminals were labeled with FM1-43 (Betz et al., 1992b) by bathing the preparation in a modified Ringer's solution (53.7 $\mathrm{mm} \mathrm{NaCl}$ and $60 \mathrm{~mm} \mathrm{KCl}$ ) containing $2 \mu \mathrm{M} \mathrm{FM1-43} \mathrm{for} 5 \mathrm{~min}$. Preparations were washed for a minimum of $30 \mathrm{~min}$ before images were captured.

Microinjection of cells. Motor nerves were filled with fluorescent dyes by ionophoretic injection through a microelectrode placed in the axon within $100 \mu \mathrm{m}$ of the last node of Ranvier of the terminal to be examined. Some impalements of the myelinated motor nerve were assisted using a piezoelectric device (Piezo Stepper P-2000; Physik Instrumente GmbH, Waldbronn, Germany). To prevent contraction when impaling motor nerves either $d$-tubocurarine chloride (10 $\mu \mathrm{M}$; Sigma, St. Louis, MO) was added to the bath or $\left[\mathrm{Ca}^{2+}\right]_{\mathrm{o}}$ was reduced from 1.8 to $0.4 \mathrm{~mm}$. TSCs were also filled with fluorescent dyes by ionophoretic injection through a sharp microelectrode, although filling often occurred without current application, which if applied, was always $<0.1 \mathrm{nA}$. Motor nerves were filled over periods of up to $15 \mathrm{~min}$, whereas TSCs were filled over periods of $<2$ min. The tapered portion of microelectrodes was filled with a $3 \mathrm{~mm}$ aqueous solution of the fluorescent dye in $140-200 \mathrm{mM} \mathrm{KCl}$, and the barrel of the microelectrode was filled with $150 \mathrm{~mm} \mathrm{KCl}$. The final electrode resistance was between 250 and $350 \mathrm{M} \Omega$.

Fluorescence microscopy. Fluorescent dyes were excited using a mercury arc lamp reflected light fluorescence attachment on a BHT series upright Olympus Optical (Tokyo, Japan) microscope. An Olympus Optical fluorescein filter set was used to view FM1-43, OG-1, OG-5N, and AF488, whereas a rhodamine filter set was used to view AF568. Photodamage was minimized by stopping down the aperture iris diaphragm and inserting a 50\% neutral density filter in the excitation light path. Fluorescence was observed through an Olympus Optical $40 \times$ water immersion objective (0.7 numerical aperture) using either a $1 / 3$ inch (model WV-BP310; Panasonic, Secaucus, NJ) or 1/2 inch (model 49125010; Cohu Inc., San Diego, CA) chip CCD camera. Pixellation of images was 7.45 or 5.83 pixels per micrometer for the cameras, respectively. Images were acquired using a Scion Corp. (Frederick, MD) LG3 frame grabber. On-chip integration (25 frames per second) greatly increased the effective light sensitivity of the Cohu Inc. camera, reducing the illumination intensity and illumination times required. Most images are the product of integrating 32 frames. Microinjection of cells and all electrophysiological measurements were accomplished on this same microscope by alternating epi-illumination using the $100 \mathrm{~W}$ mercury arc lamp with trans-illumination using a $50 \mathrm{~W}$ incandescent light source.

Processing of images to enhance their contrast has been described previously (Macleod et al., 1999). An FM1-43 blob was considered to be a discrete cluster of vesicles if it contained three contiguous pixels, each with a fluorescence value greater than two SDs above the average nonterminal (background) pixel fluorescence value. Changes in fluorescent microstructures over time were only quantified along terminal lengths in which the entire distal portion could be brought into sharp focus and did not cross the plane of focus. Where there was ambiguity about the number of discrete blobs, multiple images were captured using a number of focal planes. The same procedure was used when examining apparent changes in fluorescent structures over time to ensure that these changes were not the result of movements such as rotations of the structures.

Calcium imaging. To attain adequate fluorescence from OG-1 within motor nerve terminals during calcium imaging experiments, these dyes had to be injected for a minimum of $4 \mathrm{~min}$ with an ionophoretic driving force of up to $0.5 \mathrm{nA}$. The resting membrane potential of the axon was required to be stable and more polarized than $-60 \mathrm{mV}$ at the end of the injection. Calcium was present in the Ringer's solution at $1.8 \mathrm{~mm}$, as well as curare $(10 \mu \mathrm{M})$, to prevent contraction of the muscle. The preparation was then transferred to an organ bath, in which it could be inverted for viewing by a Leica (Wetzlar, Germany) TCS 4D laser confocal microscope. An excitation wavelength of $488 \mathrm{~nm}$ was used from an argonkrypton laser with a $515 \mathrm{~nm}$ long-pass emission filter. Images were acquired of the OG-1 fluorescence within a $20 \times 20 \mu \mathrm{m}$ area $(256 \times 256$ pixels) of the motor nerve terminal branch during stimulation of the motor nerve bundle $(20 \mathrm{~V}, 0.08 \mathrm{msec}$ pulses $)$. The area was sampled in a time of $137 \mathrm{msec}$, with a delay of $79 \mathrm{msec}$ between frames. Thirty-five consecutive frames were collected for each trial (seven prestimulation), and trials were repeated a minimum of $2 \mathrm{~min}$ apart. The time the preparation was exposed to the excitation light was kept to a minimum, and laser power was also kept to a minimum while the voltage of the photo-multiplier was maximized. Fluorescence images were analyzed using NIH Image software. Data are represented as $\Delta F / F$, where $F$ is the prestimulation level of fluorescence and $\Delta F$ is the change in the level of fluorescence. Data from consecutive trials were normalized to their prestimulation level of fluorescence before being combined to calculate an average. Quantification of the fall in the level of fluorescence during trials in which the preparation was not stimulated $(\sim 5 \%)$ was used to correct for the change in fluorescence attributable to photo-destruction of the dye alone (bleach).

Electrophysiology. Electrophysiological recordings were made using four extracellular electrodes whose tips were placed at the points of a quadrilateral straddling a nerve terminal branch. The electrode tips were at most $8 \mu \mathrm{m}$ apart. The tips of the electrodes were heat polished to a final inner diameter of $0.5-1.5 \mu \mathrm{m}$ and then filled with an aqueous solution containing $2 \mathrm{M} \mathrm{NaCl}$ and $0.4 \mathrm{mM} \mathrm{CaCl}_{2}$. The electrodes were observed through trans-illumination and positioned while the nerve terminal was below the plane of focus. The terminal was then brought into focus, and images were taken using both trans-illumination and epifluorescence, the latter being used to locate the FM1-43-stained blob relative to the electrodes. The potentials of the four electrodes were then recorded simultaneously for varying times, while any movement of the electrodes or muscle surface was monitored by a video recording of the region using a low level of trans-illumination. Bright-field and fluorescence images were again taken at the completion of recording. An Iso-DAM8A amplifier with four independent channels (World Precision Instruments, Sarasota, FL) was used with active head stages to record the potentials from the electrodes. Data were collected using a MacLab/4s (ADInstruments, Mountain View, CA) data acquisition system, low-pass filtered at $5 \mathrm{kHz}$, and digitized at $20 \mathrm{kHz}$. All negative-going events that were discernible by eye were measured using Igor Pro (WaveMetrics Inc., Lake Oswego, OR). For a set of amplitudes to be accepted as corresponding to the same quantal event, they were required to occur within $0.5 \mathrm{msec}$ of each other, and all amplitudes were required to be more than two SDs of the noise amplitude. Recordings were rejected for any one of the following reasons: changes in the bright-field or fluorescence appearance of the terminal; bursting behavior, although a consistently high level of spontaneous release was accepted; and movement of either an electrode tip or the terminal branch by $>1 \mu \mathrm{m}$ during the period of recording. Events were regarded as evoked if the beginning of the negativegoing trace was within $5 \mathrm{msec}$ of the stimulus artifact.

Determination of current source locations. Data on the positions of the microelectrode tips and the amplitudes recorded from each electrode allow the calculation of the coordinates of the most probable site of postsynaptic current generation for each event. In previous studies, three extracellular electrodes have been used to simultaneously record release from a terminal, and the current source locations have been found analytically (Zefirov et al., 1990). This approach gives, in general, two solutions for each event, one of which must be rejected. Macleod et al. (1999) used a selection criterion based on the amplitude distribution of the recorded events. In such an approach, the solution with the smallest amplitude in each pair is used to construct an approximate amplitudefrequency histogram for the "correct" solutions. This distribution is then used to discriminate between each solution in a pair. Use of a fourth intracellular electrode has demonstrated the validity of this approach, provided most of the recorded release occurs within the region straddled by the electrodes.

In recording release from the most distal blob of a terminal branch, the assumption that most of the release detected by all electrodes occurs within the electrodes cannot be justified. These sites are usually weakly stained with FM1-43 and may not even be associated with functional postsynaptic receptor sites. To ensure that release was indeed coming from the most distal blob and not a nearby blob on the main terminal branch, four extracellular electrodes were used to record release. This measure in most cases removed the ambiguity inherent in the threeelectrode technique. Use of a fourth electrode increases the spatial resolution of which the technique is capable and allows the signal-tonoise ratios of the electrodes to be taken into account. Although in the three-electrode case the two solutions are exact, the solution with four electrodes is necessarily a "best fit" to the data from each electrode. The solution can therefore be weighted with respect to the various signal-tonoise ratios.

A numerical algorithm, detailed in Appendix, was used to find the most probable site of current generation for each event. In summary, a square analysis region centered on the electrodes was divided into a grid of points. At each point, the value of a function giving the probability of 


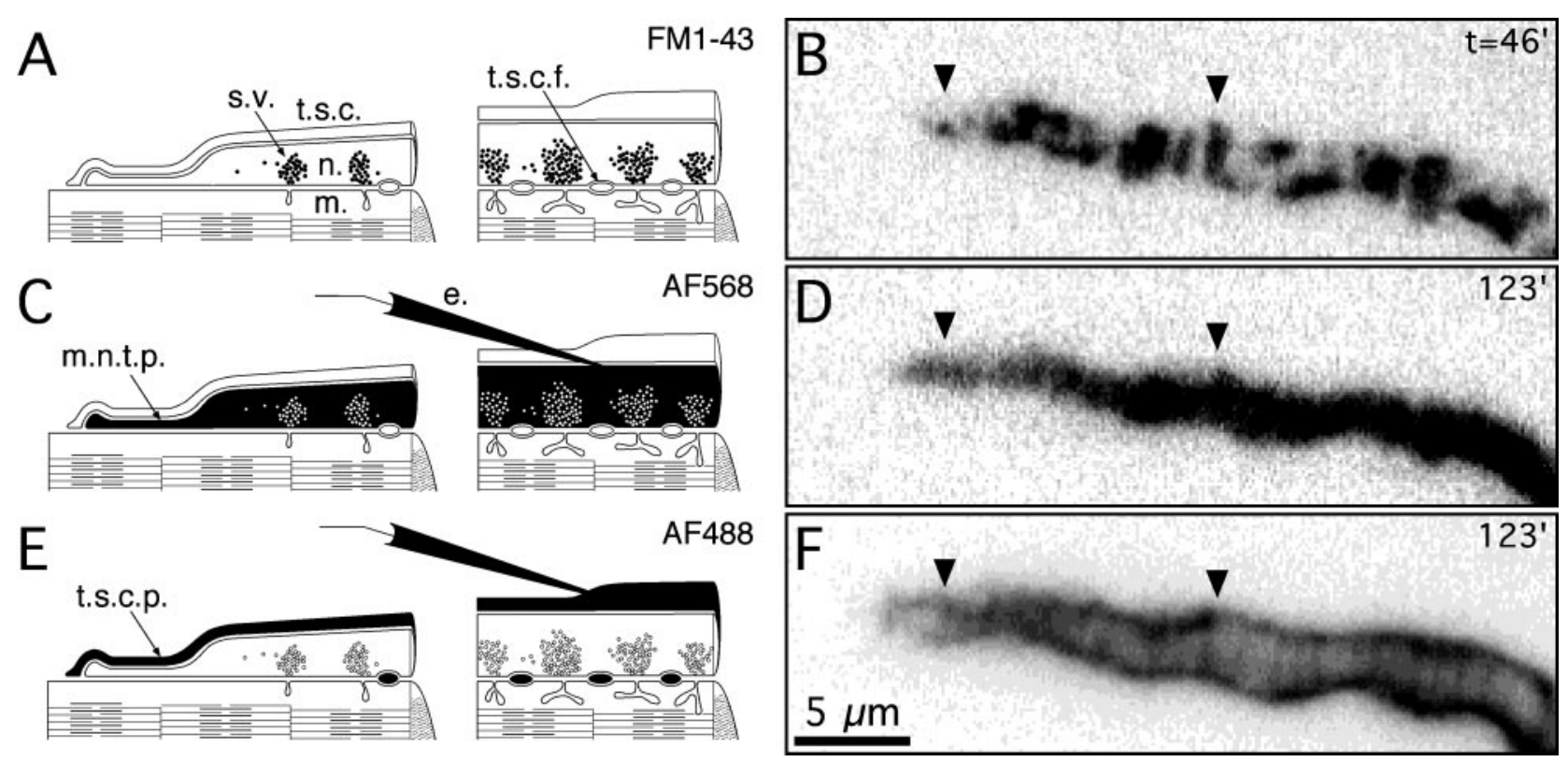

Figure 1. Labeling components of the live motor nerve terminal. $A$ is a longitudinal section representation of two noncontiguous portions of a motor nerve terminal (n.) with its clusters of synaptic vesicles (s.v.), lying on the skeletal muscle fiber $(m$.), covered by the terminal Schwann cell (t.s.c.) and clasped by the TSC fingers (t.s.c.f.). $B$ shows the distal $30 \mu \mathrm{m}$ of a live terminal branch that has been stained with FM1-43 to reveal the clusters of vesicles 46 min before the image was captured. $C$ depicts a sharp microelectrode $(e$.) injecting a dye into the nerve terminal, which sometimes reveals a motor nerve terminal process (m.n.t.p.). $D$ shows the same field of view as in $B, 74$ min after injection with AF568 and 123 min after staining with FM1-43. $E$ depicts a sharp microelectrode injecting a dye into the nuclear region of the TSC. $F$ shows the same field of view as in $B$ and $D, 3$ min after injection of the TSC with AF488 and 123 min after staining the synaptic vesicles with FM1-43. The solid arrowheads are present to assist comparison of stained elements between images $B, D$, and $F$. Time stamps represent the time, in minutes ('), since staining the preparation with FM1-43. The toad was killed 5 hr 43 min before FM1-43 staining.

release having originated there was calculated, and the coordinates of the peak of the resulting surface were taken as the position of release. In calculating this function, it was assumed that the recorded amplitude decreased as the reciprocal of distance from the recording electrode (Bennett et al., 2000a). In a minority of cases, the algorithm gave two peaks. It was decided that, in such cases, the highest peak would be accepted if its probability was more than twice that of the next highest peak. The coordinates and amplitudes of all such solutions, which could be assigned unambiguously, were recorded, and the mean and SD of the amplitudes was used to discriminate between the ambiguous solutions.

\section{RESULTS}

The work reported here depends on the injection of fluorescent dyes directly into the motor nerve terminal and TSC of single motor end plates. Any one of the fluorescent compounds AF568, AF488, or OG-5N were injected with a sharp microelectrode directly into the myelinated portion of the motor nerve or into the proximal portion of a terminal branch (Fig. $1 C$ ), resulting in the labeling shown in Figure $1 D$, and then the other fluorescent compound into the nuclear region of the TSC covering that branch (Fig. $1 E$ ), resulting in the labeling shown in Figure $1 F$. The styryl dye FM1-43 could also be used to determine the distribution of clusters of synaptic vesicles (Fig. 1A) that appeared as blobs of FM1-43 fluorescence (Fig. $1 B$ ). It was then possible with these procedures to simultaneously determine the extent of a terminal branch, together with the distribution of its synaptic vesicles and its relationship with the TSC.

\section{Rapid synapse formation and regression}

Experiments were performed to test whether new synapses can form rapidly at the end of motor nerve terminal branches. Terminal branches were stained by exposing the preparation to $2 \mu \mathrm{M}$ FM1-43 in a $60 \mathrm{~mm}\left[\mathrm{~K}^{+}\right]$Ringer's solution for $5 \mathrm{~min}$. After washing the preparation for $30 \mathrm{~min}$, the branches were examined using epifluorescence through a fluorescein filter set to determine the spatial distribution of vesicles undergoing exocytosis-endocytosis along individual terminal branches. Images were acquired to record the pattern of FM1-43 blobs within a $10 \mu \mathrm{m}$ radius of the center of the most distal FM1-43 blob. Approximately 16 (14-18) hr later, the preparation was stained again with FM1-43 using the same protocol as used earlier. The use of a long-pass emission filter reduced any ambiguity associated with the appearance of new sources of fluorescence because FM1-43 precipitate appears orange, whereas FM1-43 located in the membranes of synaptic vesicles appears yellow. Altogether, 120 terminal branches were examined on 21 iliofibularis muscles in the seasons of spring, summer, and autumn. In many cases, new yellow FM1-43 blobs were found to have formed over a $16 \mathrm{hr}$ period at the distal end of the terminal branch, either immediately adjacent to a previously existing blob (Fig. $2 A$, four branches) or $>5 \mu \mathrm{m}$ from any previously existing blob (Fig. $2 B$, two branches), or lateral to the end of the branch (Fig. $2 C$, eight branches). On three branches, new blobs had intercalated between existing blobs in the predistal region of the branch.

Regression of FM1-43 blobs also occurred in which blobs disappeared from the end of the branch (Fig. 2D,F, 16 branches), as well as from locations lateral to the end of the branch (Fig. $2 F$, three branches) or between other FM1-43 blobs in the predistal region of the branch (Fig. 2E, nine branches). Adjacent FM1-43 blobs often merged to form a single blob in the location formerly occupied by two. Less commonly, single blobs split into two discrete blobs occupying the same location as the original blob. A merger of blobs or a split of a blob occurred on branches in which 

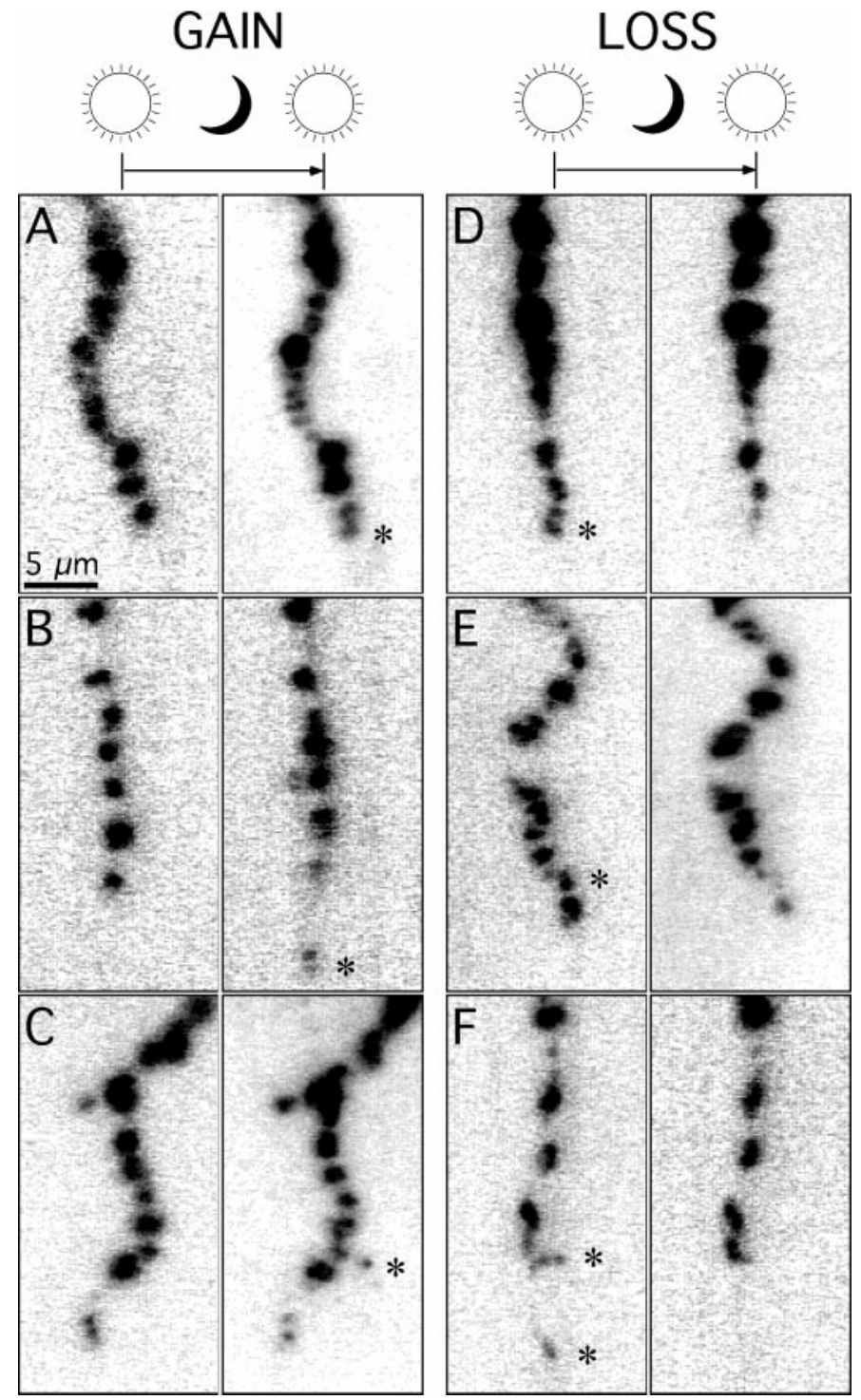

Figure 2. Individual clusters of synaptic vesicles, indicated by FM1-43 blobs, can appear or disappear from the ends of terminal branches over hours. FM1-43 blobs are shown along the distal portion of six terminal branches $(A-F)$ after an initial staining with FM1-43 (left panel), and the same terminal branches are shown after restaining $16 \mathrm{hr}$ later (right panel). $A-C$ show the appearance of new clusters of FM1-43-stained vesicles on these terminal branches (asterisk), whereas $D-F$ show the disappearance of such clusters over the same period (asterisk). The scale bar shown in $A$ is the same for all images.

either growth or regression occurred and so were classed as neither growth nor regression events and are represented separately in the summary of changes (Fig. 3).

Altogether, $41(35 \%)$ of the terminal branches showed neither a growth nor regression event as described above and no merging or splitting of FM1-43 blobs. Of all branches examined, there were no branches in which blobs were lost from one location and gained in another location on the same branch ending. Usually no more than one branch ending was examined per terminal to reduce the amount of photo-excitation within any one field of view. The histograms in Figure 3, $A$ and $B$, do not sum to $100 \%$ because some branches displayed more than one form of plasticity. Six muscles were stimulated via the nerve trunk over the $16 \mathrm{hr}$ period using either single pulses $(0.08 \mathrm{msec}, 12 \mathrm{~V})$ at $0.5 \mathrm{~Hz}$ or

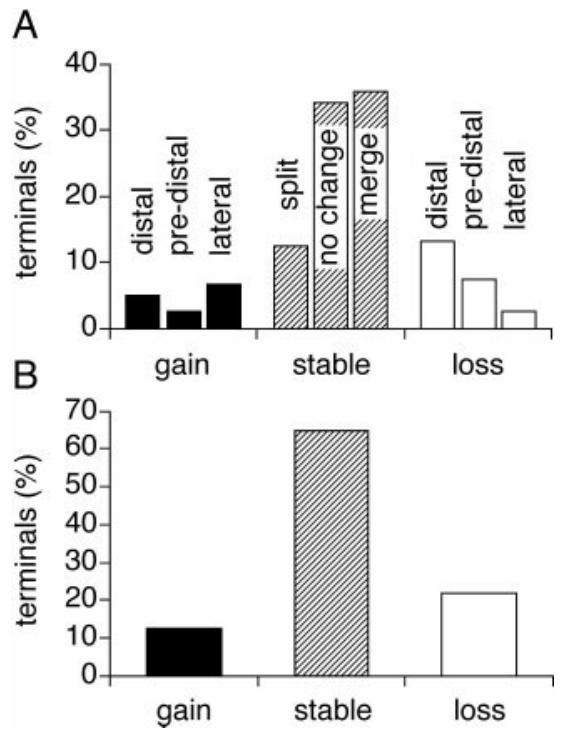

Figure 3. Histograms summarizing the changes in the pattern of FM1-43 blobs at terminal branches over a $16 \mathrm{hr}$ period. Changes were determined over the last $10 \mu \mathrm{m}$ of branch length for 120 terminals. The histogram in $A$ shows the percentage of branches in which one or more new blobs appeared ( filled columns) or were lost (open columns) from the most distal, predistal, or lateral portions of the distal length. The hatched columns represent the percentage of terminals in which preexisting blobs split, preexisting blobs merged, or there was no discernible change in the pattern of blobs. The histogram in $B$ shows the percentage of terminals in which one or more new blobs appeared (filled column), the blobs were relatively stable (hatched column; including the terminals that showed a split or merger of blobs), or one or more blobs disappeared (open column).

twin pulses ( $5 \mathrm{msec}$ delay) at $0.1 \mathrm{~Hz}$, but stimulation appeared to have little effect on the number of new synapses that appeared (five growth events on 36 terminal branches; 14\%). Growth events were observed in the presence of curare $(10 \mu \mathrm{M})$ over a 16 hr period, but the numbers were slightly less (two growth events on 36 terminal branches; $6 \%$; four muscles).

\section{The functional properties of synapses at the end of terminal branches}

To determine whether vesicle clusters at the distal ends of terminal branches constituted fully functional synapses, two kinds of experiments were performed. One series of experiments used electrophysiological techniques to determine whether neurotransmitter was being released from the most distal cluster of synaptic vesicles and whether there was a patch of postsynaptic receptors associated with it. The second series of experiments used calcium imaging techniques to determine whether the calcium indicator that filled the distal extremities of the terminal branches showed a normal influx of calcium in response to an action potential.

In the first series of experiments, FM1-43 blobs isolated on the end of terminal branches (Fig. $4 A$ ) were surrounded by an array of external electrodes (Fig. $4 B$ ). The sites of spontaneous transmitter release [miniature end-plate potentials (MEPPs)] were then ascertained with respect to the FM1-43 blob, together with the amplitude of these events, using the algorithm described in Appendix. Figure $4 C$ is a spatial plot of the source of the current for 54 spontaneous events. Only those events within the radius of detection of all electrodes can be plotted. The average amplitude of spontaneous events (corrected for spatial decay) is $0.33 \pm 0.14$ $\mathrm{mV}$ (mean $\pm \mathrm{SD}$ ), and the amplitudes do not follow a normal 

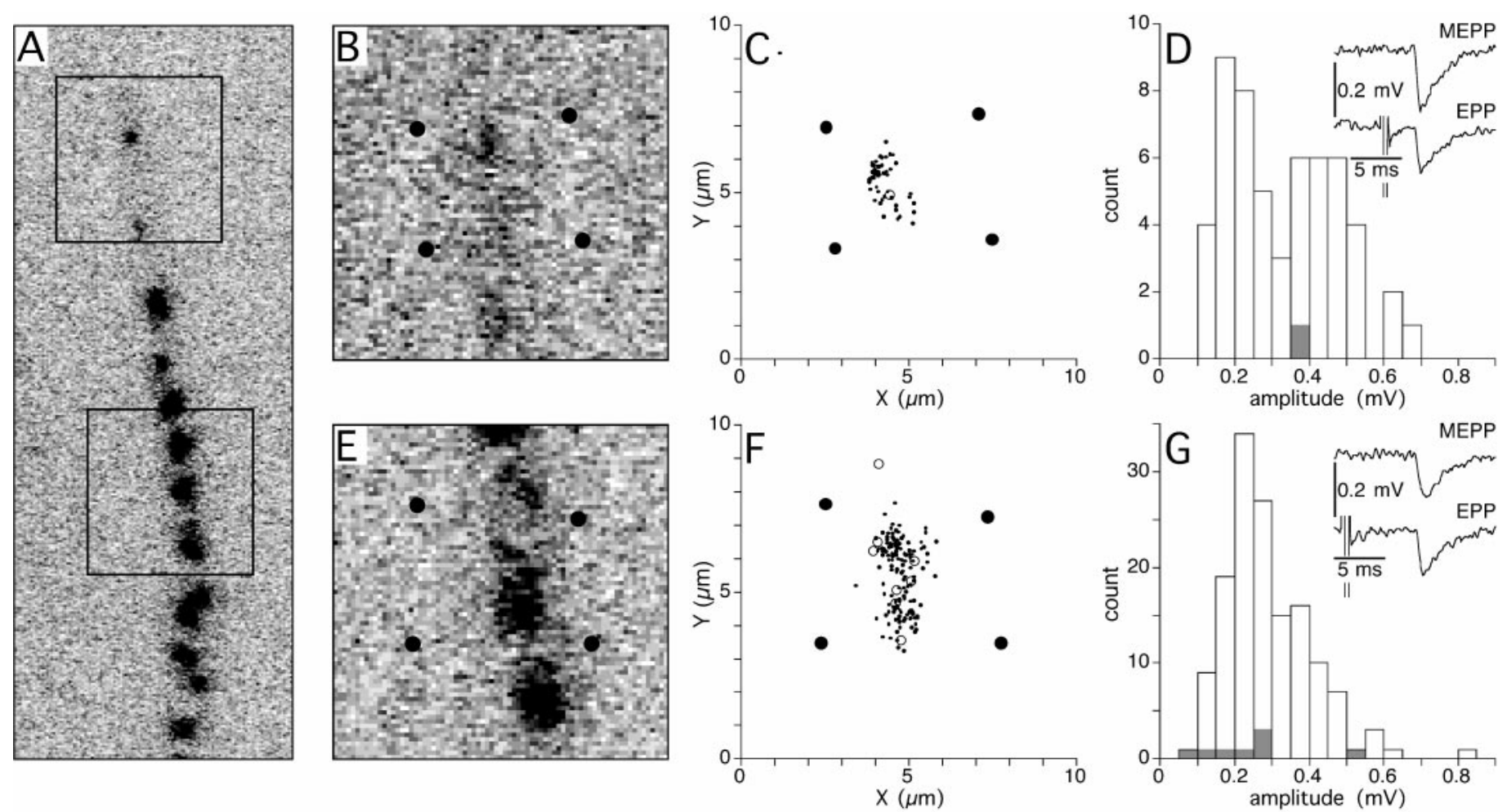

Figure 4. Signs of quantal release are detected from clusters of FM1-43-stained vesicles at the most distal end of terminal branches. $A$ shows the distal $40 \mu \mathrm{m}$ of a terminal branch that has been stained with FM1-43. Both boxed regions are $10 \times 10 \mu \mathrm{m}$. An FM1-43 blob is well separated from other FM1-43 blobs at the end of a branch. B, The top boxed region in $A$ is shown, together with the positions of four external recording electrodes ( filled circles) arranged in a rectangular array around the most distal blob. $C$ shows the positions of release of the spontaneous quanta (dots) and an evoked quantum (small open circle) detected over a 10 min period. $D$ shows an amplitude-frequency histogram for 54 spontaneous quanta (open columns) and a single evoked quantum ( filled column for the site in $C$ ). $E-G$ show a similar analysis for a 10 min recording at the more proximal boxed region in $A$. The insets in $D$ and $G$ show examples of spontaneous $(M E P P)$ and evoked (EPP) release.

distribution (Fig. 4D). The same procedure was repeated at a site $22 \mu \mathrm{m}$ more proximal on the same terminal branch (Fig. $4 E$ ). The average amplitude for the 126 spontaneous events is $0.29 \pm 0.11$ $\mathrm{mV}$, and they do follow a normal distribution (Fig. $4 G$ ). The current source locations for evoked events [end-plate potentials (EPPs)] have also been plotted at each site (Fig. 4C,F). Although the number of evoked events was low, particularly at the most distal site, their time courses were identical to the spontaneous events (Fig. 4D, $G$, traces inset). In five of six experiments, in which multiple electrodes were placed to record from the most distal blob, spontaneous release was detected within $2 \mathrm{~min}$. We were unable to ascertain further the electrophysiological characteristics of evoked release at the most distal synapses because of the very low probability of transmitter release at these synapses. Increasing $\left[\mathrm{Ca}^{2+}\right]_{\mathrm{o}}$ above $0.4 \mathrm{~mm}$ so as to increase the probability of secretion inevitably led to such a high level of cumulative quantal release from the nerve terminals that the underlying muscle fiber twitched. Such twitching resulted in displacement of the synapse relative to the array of recording electrodes, undermining the use of the algorithm to determine the location of the quantal current sources.

The question arises as to whether these distal sites display a normal calcium influx after a train of action potentials. In the second series of experiments, the motor nerve was impaled with a microelectrode and injected with the calcium-sensitive fluorescent dye OG-1 (Fig. 5A). Confocal images were then taken of the fluorescence attributable to calcium entry at both the most distal tip of terminal branches, where small varicosities could often be found (Fig. $5 B, 1,2$ ), and at more proximal portions of the terminal branch (Fig. 5B, 3-8). Nine terminal branches with one or more sites on the distal tip were examined in this way. Some distal sites (eight on six branches) did not respond during nerve stimulation at $30 \mathrm{~Hz}$ for $1 \mathrm{sec}$ (Fig. 5B, 1). Of those sites that did respond (six on six branches), the average increase in the OG-1 fluorescence was $73 \pm 25 \%$ of that observed at the more proximal portions of the terminal branch (Fig. $5 B, 2$ ). The response of those sites separated from the nonvaricose proximal portion of the branch by $>3 \mu \mathrm{m}$ (three on three branches) was $64 \pm 21 \%$. The subsequent removal of calcium from the synapses at the end of stimulation followed two exponentials, one with a time constant of $<240 \mathrm{msec}$ and the other of $>2 \mathrm{sec}$, regardless of the location at which the calcium transients were measured (Fig. $5 D$ ). The lower calcium influx at the most distal sites may explain why the probability for release of a quantum is low at such sites.

\section{Terminal Schwann cell processes}

Given that Schwann cells seem to guide the formation of nerve terminals in reinnervating muscles, as mentioned in the introductory remarks, it was of interest to establish the details of the spatial relationship between the TSCs and the nerve terminal in mature muscle. To this end, TSCs were filled with one of a number of fluorescent dyes (AF488, AF568, OG-5N, and OG-1) by placing a sharp microelectrode in the nuclear region (Fig. 1E), which could be identified in bright-field views (Fig. 6A). Some terminal branches were covered by more than one TSC, but the most distal TSC could be ascertained by identifying the most 
A

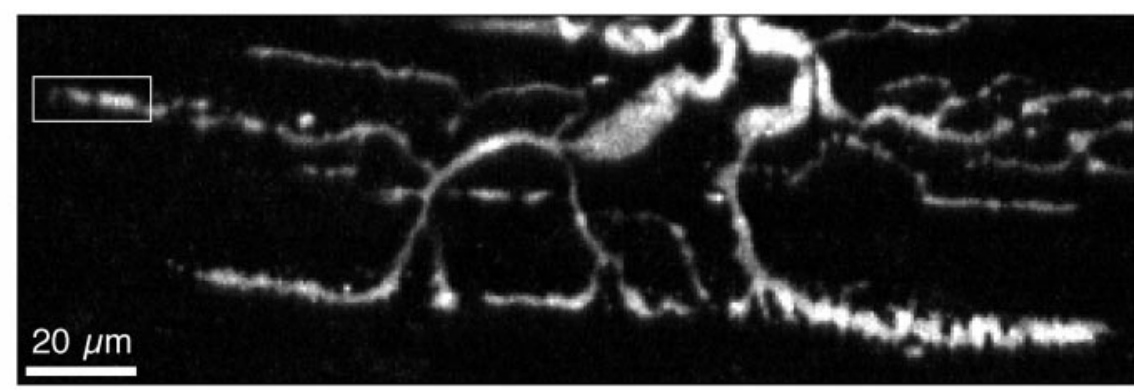

B

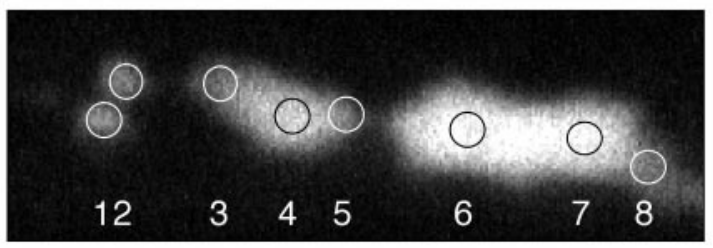

C

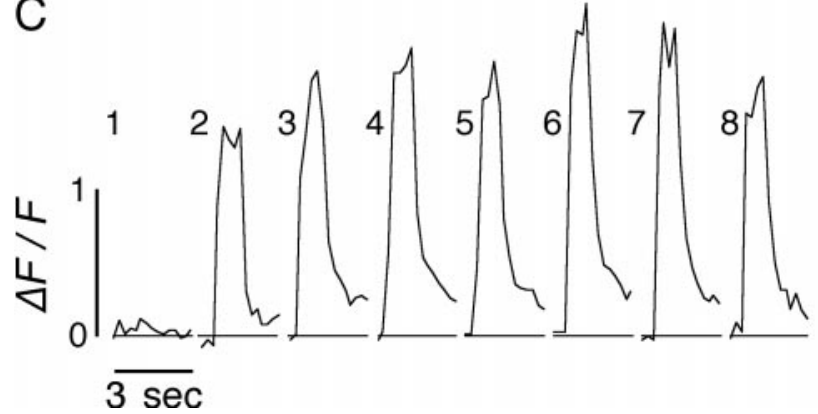

distal TSC nucleus. The body of the TSC, as well as its processes, could be easily identified (Figs. $1 F, 6 C, D$ ) after injection of the dye. In general, these processes were pseudopodial extensions of up to $10 \mu \mathrm{m}$ in length that appeared beyond the distal ends of the terminal branches, although lateral extensions were also evident (Fig. 6C,D). The distal extent of the nerve terminal was clearly determined at 24 terminal branches by injecting the nerve with a fluorescent dye. TSCs also formed fingers along the length of the terminal branches that encased the branch at regular intervals of $\sim 1-2 \mu \mathrm{m}$ (Fig. $6 C, D$ ). The spatial relationship between these TSC fingers, TSC processes, and the motor nerve terminal is indicated by the diagram of Figure $1 E$. Sixty-two TSCs were injected, allowing examination of the TSC at the distal tip of 96 terminal branches. Thirty-six of the branches had no TSC processes at the distal tip (37\%), whereas 38 had a single TSC process $(40 \%)>1 \mu \mathrm{m}$ in length, and 22 had more than one TSC process $(23 \%)$. When the TSC was injected $>16 \mathrm{hr}$ after the muscle was dissected from the animal, 11 of 18 terminal branches had more than one TSC process (61\%).

\section{The relationship between Schwann cell processes and synapses}

To determine the relationship between TSCs and the active zones of the motor nerve terminal at which synaptic vesicles cluster, the vesicles were stained with the styryl dye FM1-43 after the TSC had been injected with a fluorescent dye (Fig. 7A,B). TSC fingers can generally be seen intercalated between the clusters of vesicles. An interesting feature of the spatial relationship between the TSC and the end of the terminal branch was that the labeled pseudopodia of the former usually extended beyond the last FM1-43labeled blobs (Fig. $7 A, D, F$ ), in some cases by up to $10 \mu \mathrm{m}$ (Fig. 8, compare $A, B$ ), although this was not always the case (Fig. 8, compare the lateral blob in $A, B$ ). The concentration of the

\section{$5 \mathrm{sec}$} (14 TSCs, 21 branches).
Figure 5. Calcium influx into processes at the distal extremities of terminal branches. $A$ shows a number of motor nerve terminal branches filled with the calcium indicator OG-1 on a group of muscle fibers. $B$ is a closer view of the boxed region in $A$, which contains the most distal $20 \mu \mathrm{m}$ of a terminal branch that possesses two varicosity enlargements. Each of the circles contain a $1 \mu \mathrm{m}^{2}$ area. The level of fluorescence in the white circles $(1,2,3,5,8)$ is the same while the nerve remains unstimulated. The level of fluorescence in the black circles $(4,6,7)$ is higher than that in the white circles $(4<6=7)$. $C$, Calcium transients at the sites indicated along the terminal branch during stimulation of the motor nerve at $30 \mathrm{~Hz}$ for $1 \mathrm{sec}$. The level of fluorescence is sampled once every $216 \mathrm{msec}$ at each of the sites. $D$, Quantitative comparisons between the time courses of calcium transients from sites $1-4$ in $C$.

injected dye appears to come to an equilibrium within the entire TSC cytoplasm over a period of seconds. Forty-three terminal branches stained with FM1-43 were compared against the extent of the TSC, and TSC processes were revealed by dye injection. Without exception, the FM1-43-stained vesicle clusters at the end of terminal branches were closely associated with a TSC.

One caveat to the above observations is that fluorescence from dye in the TSC cytoplasm can persist for over $12 \mathrm{hr}$ in the absence of high levels of photo-excitation. If a fluorescent dye with the same emission spectrum as that of FM1-43 is injected before FM1-43 staining (as seen in Fig. 7A, $B$ with the injection of OG-5N), it is easy to mistake the less intense fluorescence from persistent TSC labeling for FM1-43 staining. To avoid such ambiguity, the synaptic vesicles were also stained with FM1-43 before injection of the TSC (Figs. 7C,D, 8A,B). Clearly, one would not expect to distinguish the spatial arrangement between vesicle clusters and TSC fingers in Figure 7, $C$ and $D$. Similarly, although there appears to be windows in the TSC in Figure $1 F$, they will only appear faintly because the same branch has already been stained with FM1-43, which has a similar emission spectrum to the AF488 in the TSC. The spatial relationship between the FM1-43 blobs and the TSC was most clearly delineated when the TSC was filled with a dye with a different emission spectrum to FM1-43, as shown in Figure 7, $E$ and $F$, in which AF568 was used

\section{The time course of formation and regression of Schwann cell process}

The existence of TSC processes, appearing like the pseudopodia of neuronal growth cones, which were sometimes accompanied by a motor nerve terminal process possessing an accumulation of synaptic vesicles, prompted an investigation into their lability. To this end, TSC processes were identified (Fig. 8B) and imaged at 

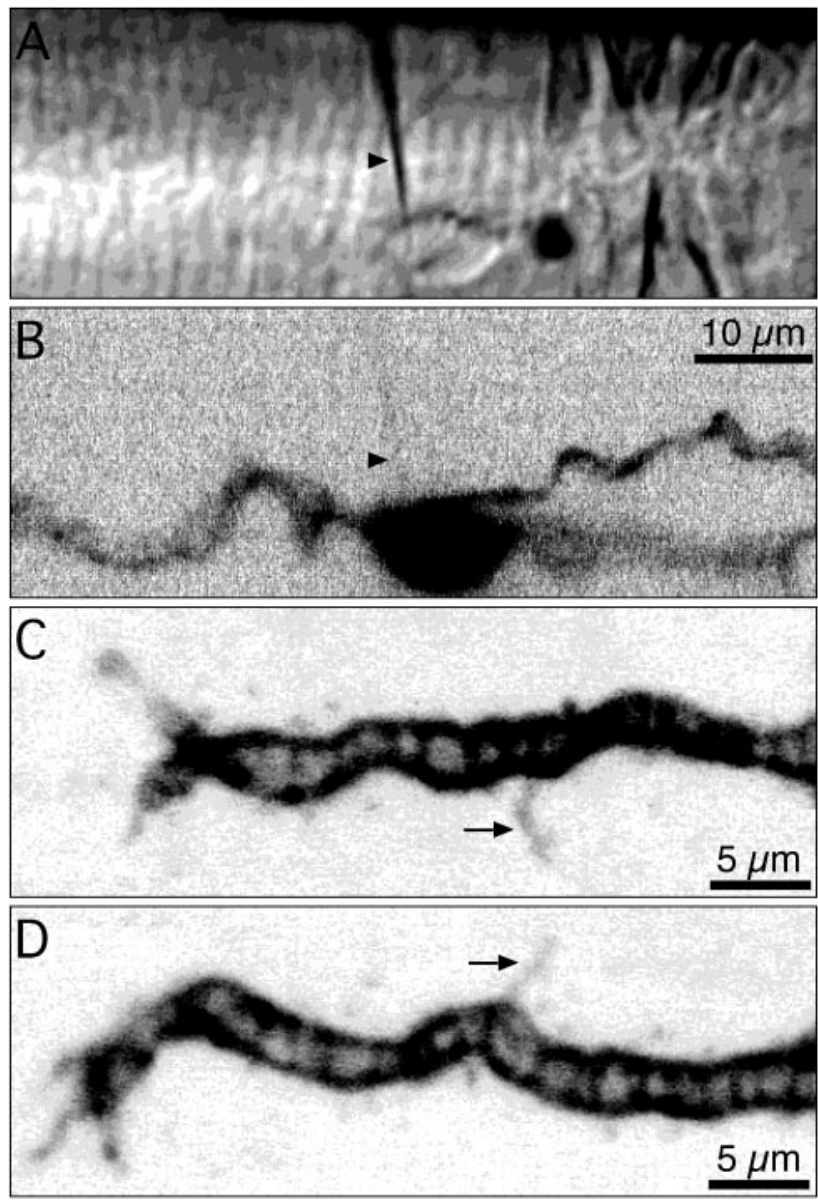

Figure 6. Processes at the end of TSCs. $A$ shows a bright-field view of the surface of a muscle fiber with a distinct TSC nucleus impaled by a microelectrode (arrowhead). $B$ shows the same field of view as in $A$ using epifluorescence to excite the OG-5N that is being injected into the TSC. $C$ and $D$ show two examples of a TSC filled with OG-5N covering the end of a terminal branch; note the TSC fingers along the length of the cell clasping the nerve terminal branch and the processes at the distal end of the branches, as well as at the side of the branches (arrows). The two branches in $C$ and $D$ are on the same TSC, on either side of the nucleus. The toad was killed $4 \mathrm{hr} 10 \mathrm{~min}$ before the TSC in $C$ and $D$ was injected. The first images of TSCs were captured within minutes of filling.

regular intervals. Some TSC processes grew a few micrometers over 5 min (Fig. 8, series in $B-D, E-G$ ). O'Malley et al. (1999) observed that intentional over-illumination of mouse TSCs stained with Calcein blue resulted in an increase in the number of TSCs in the end-plate area (detected 3-7 d later). Photoexcitation was considered unlikely to be the cause of TSC process extension in these experiments because extension did not occur in 10 of 13 cases in which the TSC processes were observed over periods of $>30 \mathrm{~min}$. An additional three TSCs, covering the distal extent of four terminal branches, were injected with dye and observed over a $16 \mathrm{hr}$ period, but no changes were observed. TSC processes could not be stimulated to extend by increasing the intensity of photo-excitation. Where multiple TSC processes were observed in the same field of view, no more than one showed extension. It was considered that damage caused by impalement and injection of the nuclear region of the cell may stimulate TSC process extension; however, because extension was limited to one TSC process, this was again considered unlikely. TSC process extension was observed with both OG-5N and AF568, making it unlikely that the type of dye injected stimulated extension.

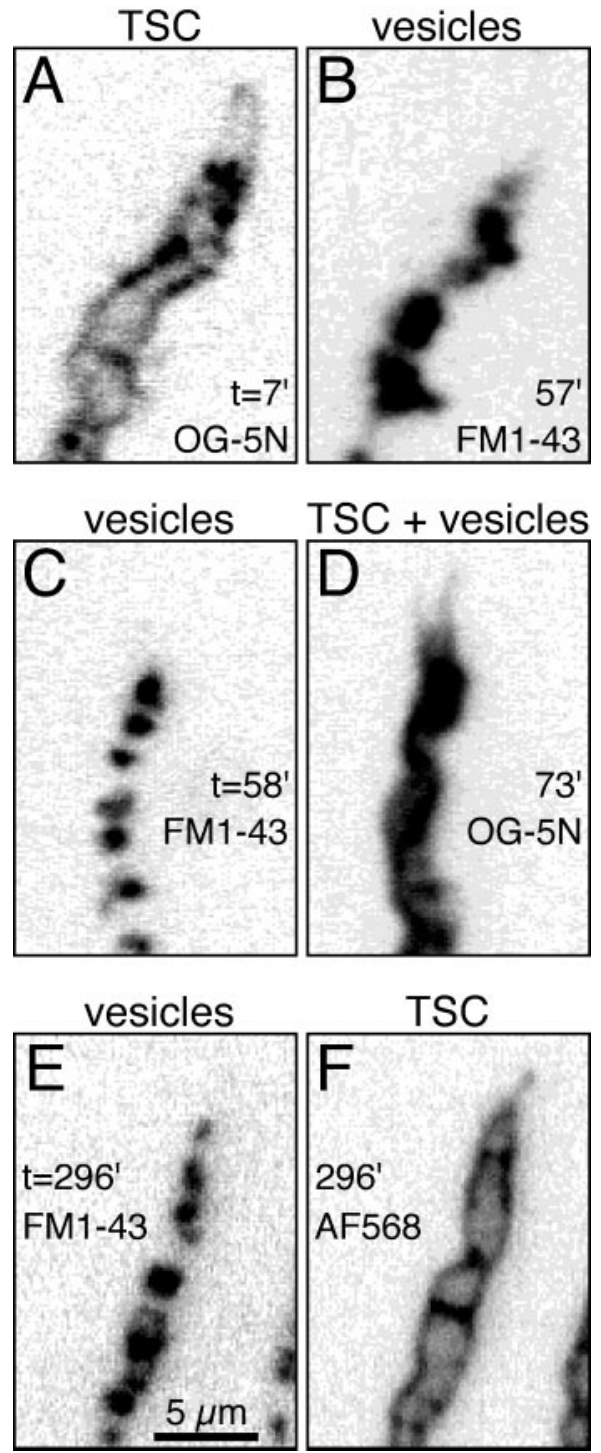

Figure 7. The relationship between TSCs and synaptic vesicle clusters. Three examples are shown, each consisting of a pair of images $(A, B ; C$, $D$; and $E, F)$; one image in the pair shows a TSC injected with a fluorescent dye (OG-5N or AF568), and the other image shows the nerve terminal branch stained with FM1-43. It should be noted that the TSC processes extend beyond the last FM1-43-stained vesicle cluster. In $A$ and $B$, the TSC was injected with OG-5N before staining the vesicles with FM1-43; in $C$ and $D$, the vesicles were stained with FM1-43 before injecting the TSC with OG-5N, and in $E$ and $F$, the vesicles were stained with FM1-43 before injecting the TSC with AF568. Time stamps represent the time since filling the TSC $(A, B)$ or the time since staining the preparation with FM1-43 $(C, D$ and $E, F)$. The first images of filled TSCs were captured $6 \mathrm{hr} 2 \mathrm{~min}(A), 8 \mathrm{hr} 31 \mathrm{~min}(D)$, and $5 \mathrm{hr} 53 \mathrm{~min}(F)$, respectively, after toads were killed. Scale bar in $E$ applies to all images.

\section{The time course of formation and regression of motor nerve terminal processes with respect to Schwann cell processes}

To investigate the possibility that new terminal processes grow at a similar rate to that of TSC processes, 24 terminal branches along with their TSCs were filled with fluorescent dyes of different emission spectra. Four terminals were followed over time using time-lapse imaging. On two terminal branches, a new terminal process formed and followed an existing TSC process over a period of $<30 \mathrm{~min}$ (Fig. $9 A, B$ ). At the remaining two terminal 

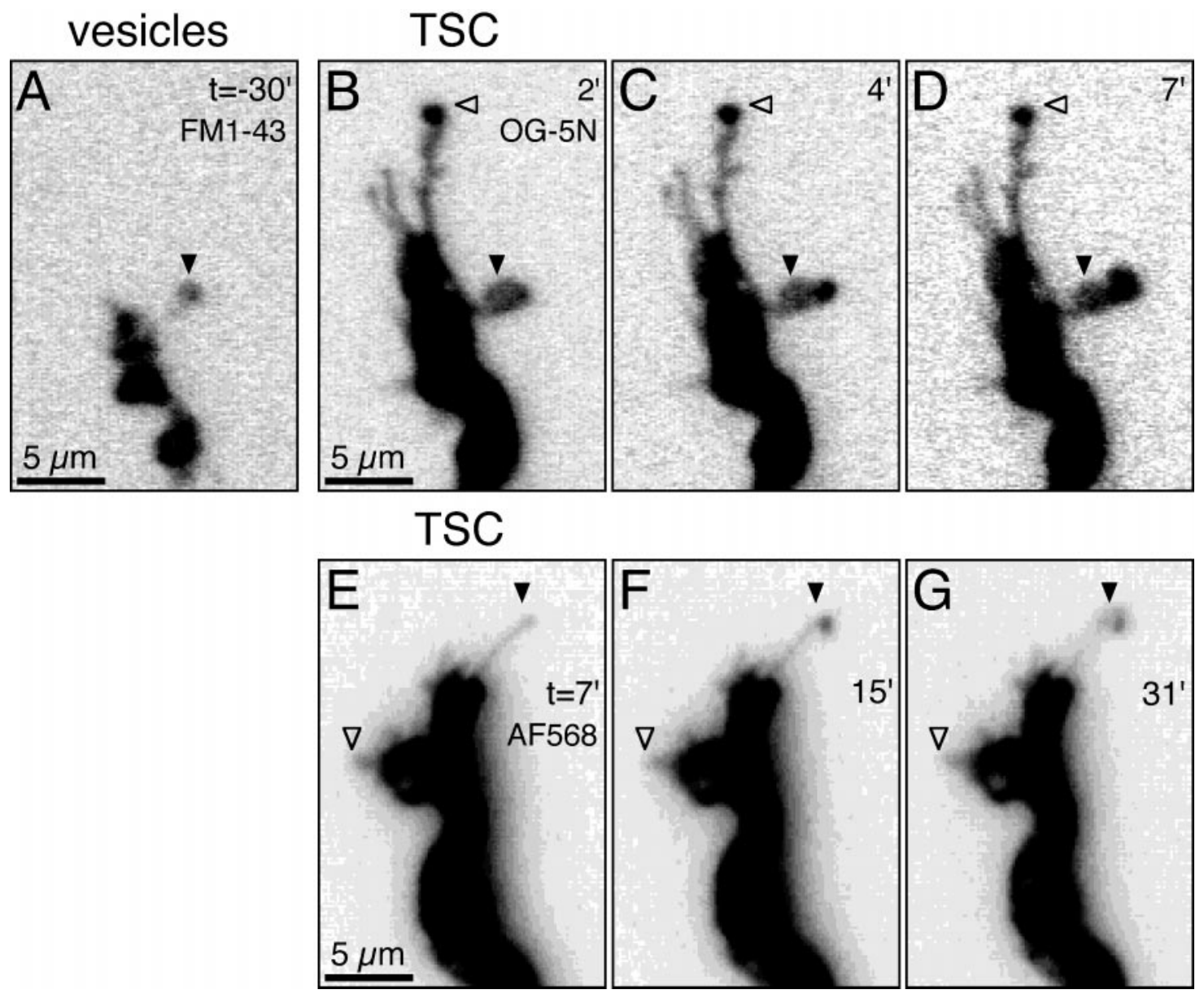

Figure 8. Individual TSC processes grow over periods of minutes. $A$ shows the end of an FM1-43-stained nerve terminal branch that possessed an isolated FM1-43 blob ( filled arrowhead) laterally removed from the long axis of the other FM1-43 blobs. $B$ shows, 2 min after injection of OG-5N into the TSC, the extent of the TSC processes at the end of the terminal branch. The position of the lateral FM1-43 blob from $A$ is indicated by a filled arrowhead. $C$ and $D$ show the relative growth of the TSC process with respect to the fixed filled arrowhead at $4(C)$ and $7(D)$ min after injection. The longest TSC process that has no associated FM1-43 blob shows no growth (open arrowhead). Another growing TSC process is shown in the image series from $E$ to $G$, but it was not established whether it coincided with an isolated FM1-43 blob. Time stamps represent the time since staining the preparation with FM1-43 $(A-D)$ or the time since filling the TSC $(E-G)$. The first images of filled TSCs were captured $8 \mathrm{hr} 47 \mathrm{~min}(B)$ and $5 \mathrm{hr} 29$ min $(E)$, respectively, after toads were killed.

branches, no TSC or TSC processes were associated with the distal tips (Fig. 10). One nerve terminal tip extended in the form of a long varicose process (Fig. 10A), and the other was blunt (Fig. $10 B$ ). These regressed over a $6 \mathrm{~min}$ and a $45 \mathrm{~min}$ period, respectively, back toward the distal margin of the Schwann cell. At the remaining 20 terminal branches, which were injected along with the TSCs, no movement of either element was observed over a minimum of $30 \mathrm{~min}$ of observation.

The features and relative positions of the ends of all of the 24 terminal branches and the TSCs at the time of injection are summarized in Figure 11. TSC processes were observed on 10 branches (Fig. $11 A, B$ ), and terminal processes were observed on five branches (Fig. 11 $A, E$ ). Four of the terminal processes were up to 5- $\mu \mathrm{m}$-long, very fine, and associated with a TSC process that exceeded them in length and breadth (Fig. 11A). The other terminal process was over 10 - $\mu \mathrm{m}$-long, with two varicosities along its length (Fig. 11E). Six branches displayed one or more TSC process without associated terminal processes (Fig. 11B). The remaining 13 branches showed no processes of any sort, and the end of the nerve terminal branch either coincided with the extent of the TSC (Fig. 11C, 11 branches) or exceeded it by one to several micrometers (Fig. 11D, two branches).

\section{DISCUSSION}

The styryl dye FM1-43 has been used in this study to label vesicle pools that are normally found in conjunction with one or two active zones at amphibian motor nerve terminals (Betz and Bewick, 1992; Macleod et al., 1999). The question arises as to the applicability of this technique to identify newly forming synapses at the distal extremities of remodeling motor nerve terminals. Blobs of FM1-43 staining are known to align with the postsynaptic acetylcholine receptor clusters and, in general, to persist for many hours (Betz et al., 1992b; Wu and Betz, 1999), with the dye only disappearing if released into the bathing medium during destaining (Henkel et al., 1996). The size of these FM1-43 blobs is correlated with the quantal content of the EPP, as well as with the probability of quantal release at mature synapses (Macleod et al., 1998; Kuromi and Kidokoro, 1999; Quigley et al., 1999). However, the FM1-43 blobs are labile during nerve stimulation, with some of them coalescing (Betz et al., 1992a). The coalescence identified here is not attributable to spontaneous impulse activity because such activity was not detected in the motor nerves, even after 16 hr in vitro.

Quantal secretion at individual synapses of amphibian motor 

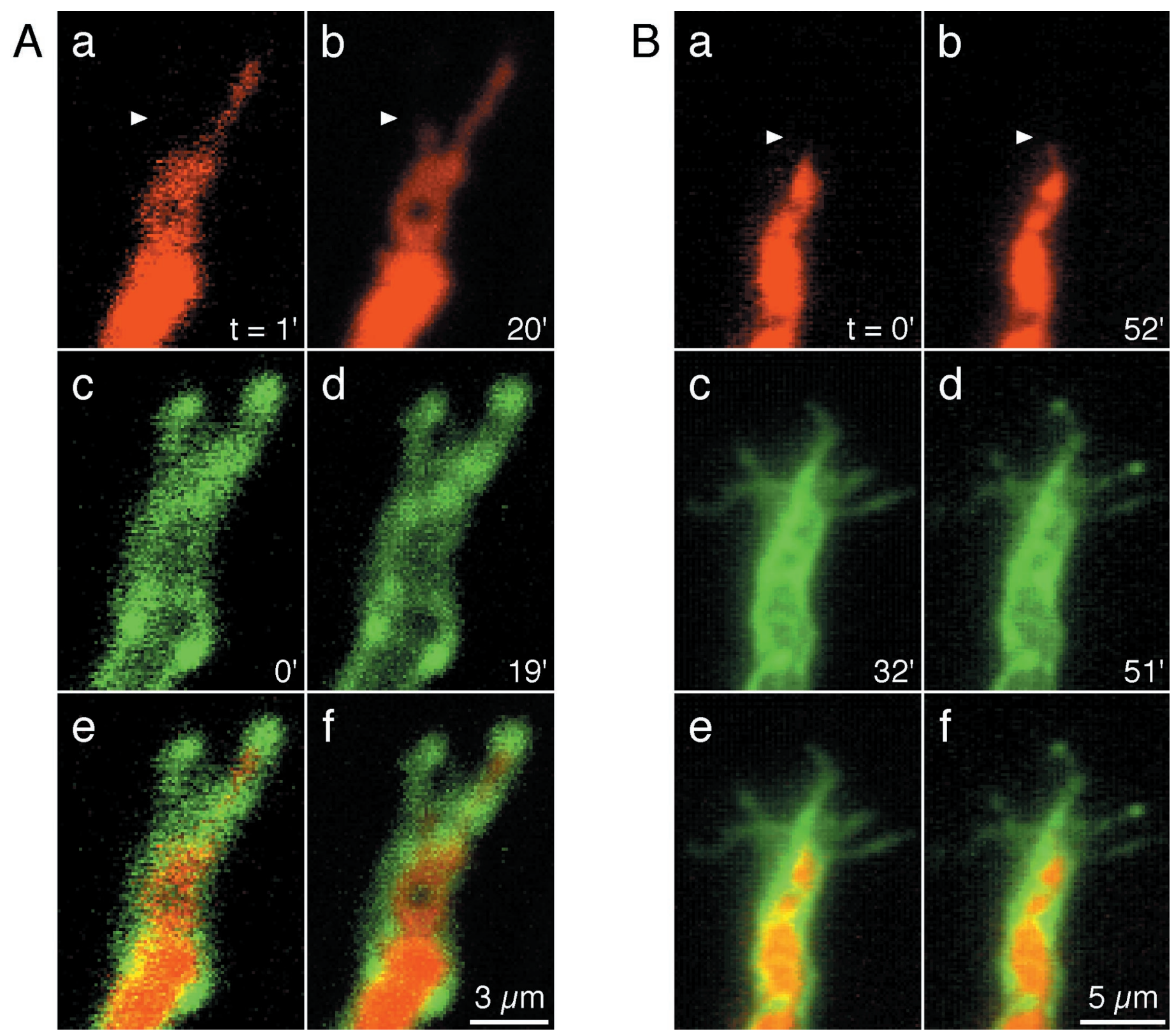

Figure 9. The growth of motor nerve terminal processes with respect to TSC processes. Shown are the changes in terminal processes $(a, b)$, as well as their associated TSC processes $(c, d)$ over a time interval of $19 \mathrm{~min}$ in $A$ and $52 \mathrm{~min}$ in $B$. In each case, $e$ and $f$ give the superimposed images. In $A$, a small terminal process appears between $a$ and $b$, and this follows the left-hand TSC process that has remained stationary over this time, as shown by $c$ and $d$. In $B$, a small terminal process appears between $a$ and $b$, and this follows the central TSC process that shows little change over the time between $c$ and $d$. In $A$, OG-5N was injected into the nerve terminal and AF568 into the TSC. In $B$, AF488 was injected into the nerve terminal and AF568 into the TSC. Time stamps represent the time since capturing the first images of the nerve and TSC in the before-after series. The first images of filled TSCs were captured $5 \mathrm{hr} 16 \mathrm{~min}(A c)$ and $3 \mathrm{hr} 43 \mathrm{~min}(B c)$, respectively, after toads were killed.

nerve terminals can be resolved using an array of external microelectrodes placed about individual active zones, which provides a spatial resolution for delineating the sources of quantal release of $\sim 250 \mathrm{~nm}$ (Zefirov et al., 1990; Macleod et al., 1999; Bennett et al., 2000a), which is at the limits of the resolving power of the light microscope. This approach opens up the possibility of recording quantal secretion from newly formed synapses that are displaced only a few micrometers from established synapses. The present observations show that the most distal clusters of synaptic vesicles, revealed through FM1-43 staining, secrete transmitter onto a functional receptor patch. It was possible in only one case to obtain enough data to draw up an amplitude-frequency histo- gram of spontaneous releases, and these did not follow a Gaussian distribution. Such a result is typical of early forming synapses during development of amphibian neuromuscular junctions (Bennett and Pettigrew 1975). Zefirov and Cheranov (1995) used three external electrodes to record and locate the electrical signs of transmitter release at the ends of nonvisualized amphibian motor nerve terminal branches. Although they were unable to determine unequivocally the distal extent of the terminal branch that they were recording from, their observations did show a lower probability of transmitter release from the last active zone of the mature terminal branch.

During development of end plates, FM1-43-stained clusters of 


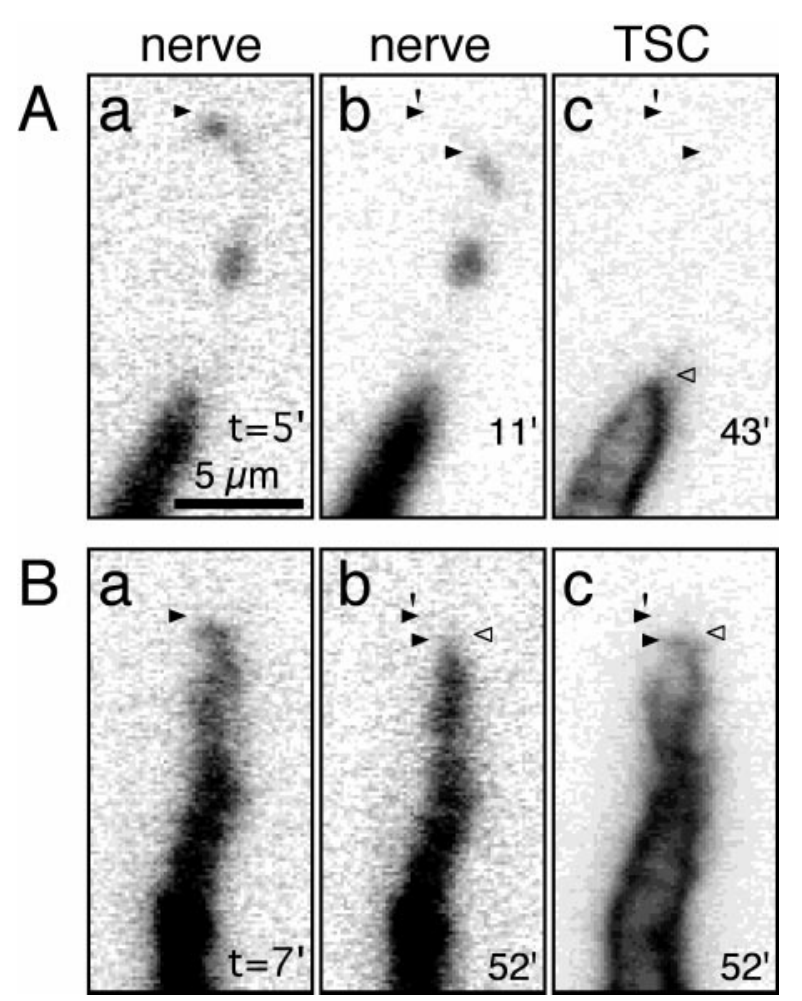

Figure 10. The regression of motor nerve terminal processes with respect to the TSC. Shown are the changes in terminal processes $(a, b)$ over a time interval of $6 \mathrm{~min}$ in $A$ and $45 \mathrm{~min}$ in $B$. In $A$, a long terminal process, well beyond reach of the TSC and not associated with any TSC processes, regresses between $a$ and $b$. In $B$, the distal tip of a terminal branch regresses between $a$ and $b$. The TSC revealed at the end of this period has no processes and is short of the initial extent of the distal tip of the terminal. In $A$, AF488 was injected into the nerve terminal and AF568 into the TSC. In $B$, AF568 was injected into the nerve terminal and AF488 into the TSC. Time stamps represent the time since starting to inject the motor nerve with fluorescent dye. The first images of filled TSCs were captured $4 \mathrm{hr} 35 \mathrm{~min}(A c)$ and $7 \mathrm{hr} 46 \mathrm{~min}(B c)$, respectively, after toads were killed.

mobile synaptic vesicles can be observed along the length of neurites that have not contacted a target and formed synapses, although when they do so, the clusters become immobilized (Dai and Peng, 1998). These mobile clusters of synaptic vesicles have been shown recently to be associated with major cytoplasmic and membrane-associated protein precursors of the presynaptic active zone (Ahmari et al., 2000), and it is likely that they can secrete transmitter (Liou et al., 1999). This suggests that release sites may be established rapidly within newly formed processes of terminal branches, as indicated by the present observations. Changes of a comparable time course are reported from the other side of the synaptic cleft, in which a rapid turnover of postjunctional receptor proteins has been observed over hours within cultured hippocampal neurons (Okabe et al., 1999). The present work suggests that receptors are present beneath newly formed FM1-43 blobs.

The calcium influx at newly formed vertebrate peripheral synapses during normal development can be ascertained using calcium imaging techniques. It has been shown that terminals formed just a few hours previously can sustain a calcium influx in response to a nerve impulse, as well as secrete quanta (Lin et al., 1996). In the invertebrate nervous system, an initial contact between neurites and muscle cells leads to an increase in the calcium concentration in the growth cone of the neurite, which is

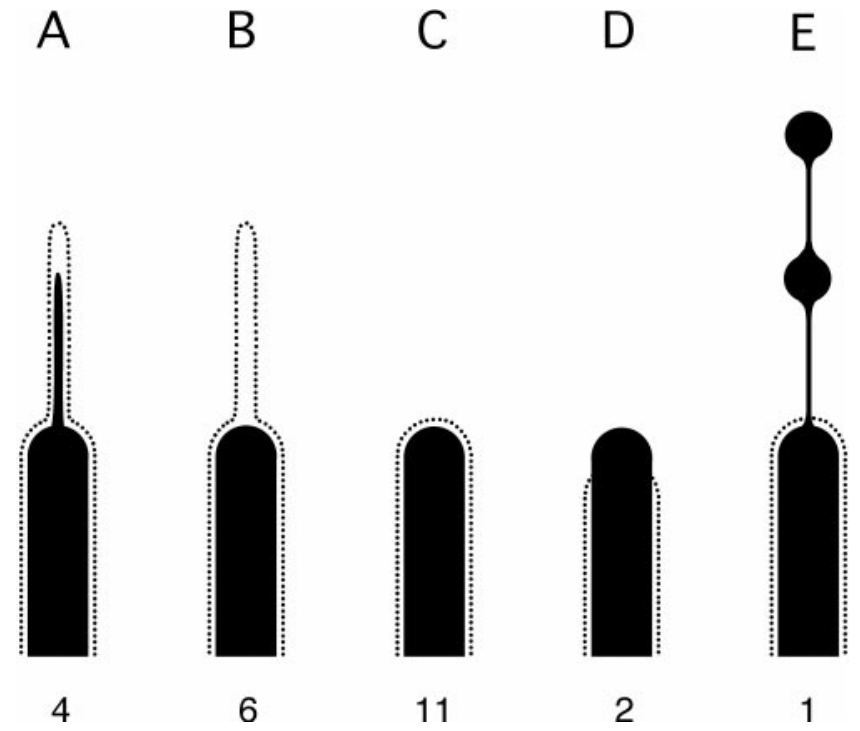

Figure 11. Static observations of the ends of motor nerve terminal branches relative to TSCs. $A-E$ are schematic representations of the different types of terminal branch endings ( filled) relative to the TSC (dotted outline). Twenty-one terminal branches filled with AF488, AF568, OG-5N, or OG-1 were compared with their overlying TSC by injecting the TSC with a fluorescent dye of a different emission spectrum (either AF488 or AF568). The number of terminal branches described by the representations, on the initial viewing, are indicated by the number below each. The scale of the features is consistent within the diagram with the terminal branch proper being $\sim 2 \mu \mathrm{m}$ in diameter.

followed by elaboration of an active zone and functional quantal secretion (Zoran et al., 1993). Shortly after amphibian motor nerve terminals first form, the $10 \mathrm{~nm}$ intramembraneous particles, which characterize the two double-particle rows that identify the mature active zone at the ultrastructural level, are found scattered in the terminal and only line up into rows at a later stage (Ko, 1985). Signs of vesicle exocytosis are evident at these primitive synapses even before these $10 \mathrm{~nm}$ particles, which are thought to be voltage-dependent calcium channels (Llinas et al., 1981), have formed two double rows (Ko, 1984). We observed calcium transients at the distal extremities of terminal branches in response to nerve stimulation that were significantly less than those that occur at the more mature synapses in more proximal parts of the terminal. It is known that different kinds of voltage-dependent calcium channels are present in synapses as they mature (Harrold et al., 1997). Part of the difference in calcium influx at any newly formed synapses (compared with mature synapses) might be attributable to the differences in calcium channel type rather than in the number of calcium channels present. It is likely that the very low probability of both spontaneous and evoked quantal release at newly formed synapses is attributable to multiple factors. Such factors include relatively low calcium influx coupled with a lack of strategic alignment of the synaptic vesicles and exocytotic protein machinery into compact units with a calcium channel, as would be necessary for efficient evoked quantal transmitter release (Rettig et al., 1997; Neher, 1998; Bennett et al., 2000b,c).

It may be argued that this work is essentially about nerve terminal degeneration because the study involves isolation of a nerve-muscle preparation and therefore severing the nerve supply of the muscle. Neurotransmission continues at the frog sartorius muscle after lesion of the sciatic nerve for $\sim 5 \mathrm{~d}$ in vivo at $20^{\circ} \mathrm{C}$ (Birks et al., 1960). When the nerve to the frog cutaneous pectoris 
muscle is cut in situ 2-3 mm from the muscle, neurotransmission remains "normal" for the first $36 \mathrm{hr}$ but fails by $48 \mathrm{hr}$ at $22^{\circ} \mathrm{C}(\mathrm{Ko}$, 1981). The ultrastructure of the end plate in the period between nerve lesion and neurotransmission failure cannot be discriminated from that of end plates without nerve lesion (Birks et al., 1960; Ko, 1981). In our preparations, the nerve was cut at 5-10 $\mathrm{mm}$ from the muscle so we would expect these end plates to remain functional $\left(\right.$ at $18^{\circ} \mathrm{C}$ ) for a period of time longer than the period over which our studies were conducted. Of course, the lack of an intact vasculature might militate against the perfused preparation being regarded as normal, and this might reduce the period of vitality. Although the frequency of synapse loss at the end of terminal branches was high over a $16 \mathrm{hr}$ period in this study ( $21 \%$ of all terminal branches), the frequency of synapse gain was also substantial (12\%). Previous studies on the frog motor nerve terminal demonstrated that synapse regression is common in frog muscles in which no intervention has taken place (Herrera et al., 1990; Chen et al., 1991). Prof use motor nerve sprouting has also been demonstrated in frog muscles in response to paralysis with TTX (Diaz and Pécot-Dechavassine, 1989) or curare (Wernig et al., 1980; Wernig and Herrera, 1986). There is no obvious reason why synapse gain and regression does not balance in this study, and we can only speculate that the isolated perfused musclenerve preparation is no longer exposed to the compounds that contribute to the maintenance of a constant level of synaptic contact.

Visualization of fine living terminal processes such as lamellipodia and filopodia can be problematic because they do not contain mitochondria (Robbins and Polak, 1988), so vital dyes such as 4-(4-(diethylamino)styryl)- $N$-methylpyridinium iodide, 3,3'-diethyloxadicarbocyanine iodide, and perhaps even Calcein blue will not reveal them. Similarly, the smallest processes do not contain vesicles or neurofilaments, so antibodies that bind neurofilaments and styryl dyes, such as $N$-(3-triethylammoniumpropyl)4-(4-(4-(diethylamino)phenyl)butadienyl)pyridinium dibromide and FM1-43, which label recycling vesicles, will not detect the processes. The fine processes of TSCs are also thought to be devoid of such microstructures. Fluorescently labeled toxins and antibodies that bind surface ligands of living motor nerve terminals (Hill and Robbins, 1991) and TSCs (Astrow et al., 1998; Georgiou and Charlton, 1999) are more powerful tools but do not allow discrimination between individual cells of the same type, and there is always the risk of nonspecific staining. For these reasons, the technique of filling the intracellular volume of both the motor nerve terminal and TSC with a nontoxic fluorescent dye has been used in this study, allowing visualization of the smallest processes, unambiguous identification of the parent cell for even the longest processes, and eliminating the risk of nonspecific or uneven staining. The possibility that these processes of motor nerve terminals only arise as a consequence of injecting the terminal with a particular dye was checked by using a variety of dyes (OG-5N, AF488, and AF568). Furthermore, such signs of plasticity were also evident in FM1-43-labeled terminals, in which dye injection is not involved. Direct injection of fluorescent dyes has here revealed fine motor nerve terminal processes and TSC processes up to $10-\mu \mathrm{m}$-long at the ends of some terminal branches. Labeling the cytoplasm of the two cell types with different dyes allows clear definition of the spatial disposition of the two living cells, which has been problematic to date. It reveals the rapid formation and regression of synapses of the kind revealed recently for hippocampal neurons in culture (Friedman et al., 2000).
Profuse motor nerve sprouting occurs in frog muscles in response to paralysis with many agents, including TTX (Diaz and Pécot-Dechavassine, 1989), botulinum toxin (Diaz et al., 1989), or curare (Wernig et al., 1980; Wernig and Herrera, 1986). Nerve sprouting, along with retraction, also occurs in the absence of such interventions over periods of months (Herrera et al., 1990; Chen et al., 1991). Using labeled peanut agglutinin (PNA) and repeated in vivo observations of frog motor nerve terminals, synaptic ECM was identified along tracts that were subsequently occupied by terminal branches (Chen et al., 1991; Chen and Ko, 1994; Ko and Chen, 1996). The possibility was raised that TSC processes precede nerve terminal growth, and the presence of these TSC processes either give the appearance of synaptic ECM, or more interestingly, actually cause differentiation of the ECM. This hypothesis relied on observations separated by long intervals but was supported by histological examination of the postsynaptic membrane. The TSCs could not be observed directly on the living muscle. In this study, the observation of TSC processes out in front of not only the most distal FM1-43 blobs but also the distal tip of the terminal suggests that TSC processes could conceivably lead the nerve terminal in growth. The growth of nerve processes along TSC processes lends strong support to this hypothesis (Fig. 11). In the studies of Ko and colleagues (Chen et al., 1991; Chen and Ko, 1994; Ko and Chen, 1996), PNA-stained ECM was never observed to be shorter than the nerve processes, making it unlikely that the synaptic ECM directs nerve process retraction. Here, TSC processes have been observed shorter than the nerve processes, suggesting that TSCs may also direct retraction at mature motor nerve terminals.

An observation of significant interest, when considering the role of the TSC at the nerve terminal, is the presence of the TSC at every release site. There are examples of the tips of nerve terminals without a covering TSC; however, at 43 terminal branches in which FM1-43-stained release sites were compared directly with the location of the dye-injected TSC, no examples could be found of a release site without a TSC in intimate contact. This observation implies a role for the Schwann cell in the formation and maintenance of release sites.

\section{APPENDIX}

The positions of the four electrodes in an $x, y$ plane are measured as $E_{0}\left(x_{0}, y_{0}\right), E_{1}\left(x_{1}, y_{1}\right), E_{2}\left(x_{2}, y_{2}\right)$, and $E_{3}\left(x_{3}, y_{3}\right)$. For a single event, the amplitudes recorded from the electrodes $E_{0}, E_{1}, E_{2}$, and $E_{3}$ are, respectively, $a_{0}, a_{1}, a_{2}$, and $a_{3}$. The recordings $a_{n}$ each have an associated error $s_{n}$.

We construct a grid of points in the $x, y$ plane that contains the electrodes and is large enough to include the release sites of all of the events. In practice, the grid is a square of side 10 or $15 \mu \mathrm{m}$. At each point of the grid $P(x, y)$, we calculate a function that gives the likelihood of release having originated at that point, given the particular recorded amplitudes. Assuming that release did occur at the point, we calculate the absolute amplitude at each of the four electrodes, $\alpha_{0}, \alpha_{1}, \alpha_{2}$, and $\alpha_{3}$. These are given by:

$$
\begin{aligned}
\alpha_{n} & =a_{n} \times \operatorname{dist}\left(P E_{n}\right) \\
& =a_{n} \sqrt{\left(x-x_{n}\right)^{2}+\left(y-y_{n}\right)^{2}},
\end{aligned}
$$

and the errors $\sigma_{n}$ are

$$
\begin{aligned}
\sigma_{n} & =s_{n} \times \operatorname{dist}\left(P E_{n}\right) \\
& =s_{n} \sqrt{\left(x-x_{n}\right)^{2}+\left(y-y_{n}\right)^{2}} .
\end{aligned}
$$



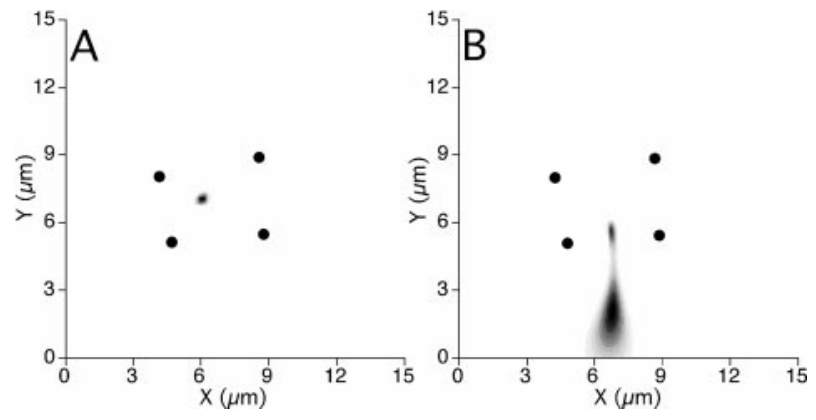

Figure 12. Grayscale representations of the probability of release. $A$ shows the solution for an event in which the position of release may be assigned unambiguously; in $B$, two peaks exist with (approximately) equal probability.

We define $\alpha$ as the weighted mean of the four measurements $\alpha_{0}$, $\alpha_{1}, \alpha_{2}$, and $\alpha_{3}$ :

$$
\begin{aligned}
& \alpha=\frac{\sum_{n=0}^{3} \frac{\alpha_{n}}{\sigma_{n}{ }^{2}}}{3} \\
& \sum_{n=0} 1 / \sigma_{n}{ }^{2} \\
& =\frac{\sum_{n=0}^{3} \frac{a_{n}}{s_{n}^{2} \sqrt{\left(x-x_{n}\right)^{2}+\left(y-y_{n}\right)^{2}}}}{\sum_{n=0}^{3} 1 /\left(s_{n}{ }^{2}\left(\left(x-x_{n}\right)^{2}+\left(y-y_{n}\right)^{2}\right)\right)} .
\end{aligned}
$$

We can find out how well $\alpha$ "agrees" with our measurements $a_{n}$ by using $\alpha$ as the argument in a (unnormalized) Gaussian with mean $\alpha_{n}$ and variance $\sigma_{n}{ }^{2}$. We shall call this function $F_{n}(x, y)$ :

$$
F_{n}(x, y)=\exp \left(\left(\alpha-\alpha_{n}\right)^{2} / \sigma_{n}\right) .
$$

The "likelihood" of release from any point $P(x, y)$ is then the product of $F_{n}$ over $n$ :

$$
F(x, y)=\prod_{n=0}^{3} \exp \left(\left(\alpha-\alpha_{n}\right)^{2} / \sigma_{n}\right) .
$$

If at $P$ the absolute amplitudes at each electrode are identical, $F$ will take its maximum value of 1 . Disagreement between the absolute amplitudes will be reflected in a value of $F$ that is $<1$.

The maximum of this function gives the most likely site of release, and the value at maximum is a measure of the agreement between the amplitudes from each trace. In most cases, $F(x, y)$ has a single peak in the analysis region (Fig. 12A). In cases in which there are more than one peak (Fig. 12B), the peak with highest probability is taken to be the "correct" solution if its peak probability is more than twice that of the next highest peak. During the analysis of a record, the position and absolute amplitude of all events for which a correct solution may be assigned are recorded. From these data, the mean and SD of the absolute amplitudes are calculated. The ambiguous solutions are then reanalyzed as follows.

The two highest peaks in $F$ have probabilities $m_{1}$ and $m_{2}$ and absolute amplitudes $\beta_{1}$ and $\beta_{2}$. The unambiguous solutions have amplitudes with mean $\bar{\alpha}$ and variance $\hat{\alpha}^{2}$. The peak probabilities are scaled to take account of the measured amplitude distribution. The adjusted peak probabilities are:

$$
\begin{aligned}
& M_{1}=m_{1} \exp \left(\left(\beta_{1}-\bar{\alpha}\right)^{2} / \hat{\alpha}\right), \\
& M_{2}=m_{2} \exp \left(\left(\beta_{2}-\bar{\alpha}\right)^{2} / \hat{\alpha}\right) .
\end{aligned}
$$

If $M_{2} / M_{1}>2$, peak 2 is accepted; if $M_{2} / M_{1}<1 / 2$, peak 1 is accepted. If neither of these conditions are met, both solutions are rejected.

\section{REFERENCES}

Ahmari SE, Buchanan J, Smith SJ (2000) Assembly of presynaptic active zones from cytoplasmic transport packets. Nat Neurosci 3:445-451.

Anzil AP, Bieser A, Wernig A (1984) Light and electron microscopic identification of nerve terminal sprouting and retraction in normal adult frog muscle. J Physiol (Lond) 350:393-399.

Astrow SH, Qiang H, Ko CP (1998) Perisynaptic Schwann cells at neuromuscular junctions revealed by a novel monoclonal antibody. J Neurocytol 27:667-681.

Balice-Gordon RJ, Lichtman JW (1990) In vivo visualization of the growth of pre- and postsynaptic elements of neuromuscular junctions in the mouse. J Neurosci 10:834-855.

Bennett MR, Pettigrew AG (1975) The formation of synapses in amphibian striated muscle during development. J Physiol (Lond) 252:203-239.

Bennett MR, Karunanithi S, Lavidis NA (1991) Probabilistic secretion of quanta from nerve terminals in toad (Bufo marinus) muscle modulated by adenosine. J Physiol (Lond) 433:421-434.

Bennett MR, Farnell L, Gibson WG, Macleod GT, Dickens P (2000a) Quantal potential fields around individual active zones of amphibian motor-nerve terminals. Biophys J 78:1106-1118.

Bennett MR, Farnell L, Gibson WG (2000b) The probability of quantal secretion near a single calcium channel of an active zone. Biophys $\mathbf{J}$ 78:2201-2221.

Bennett MR, Farnell L, Gibson WG (2000c) The probability of quantal secretion within an array of calcium channels of an active zone. Biophys J 78:2222-2240.

Betz WJ, Bewick GS (1992) Optical analysis of synaptic vesicle recycling at the frog neuromuscular junction. Science 255:200-203.

Betz WJ, Bewick GS, Ridge RMAP (1992a) Intracellular movements of fluorescently labeled synaptic vesicles in frog motor nerve terminals during nerve stimulation. Neuron 9:805-813.

Betz WJ, Mao F, Bewick GS (1992b) Activity-dependent fluorescent staining and destaining of living vertebrate motor nerve terminals. J Neurosci 12:363-375.

Birks R, Katz B, Miledi R (1960) Physiological and structural changes at the amphibian myoneural junction, in the course or nerve degeneration. J Physiol (Lond) 150:145-168.

Chen L, Ko CP (1994) Extension of synaptic extracellular matrix during nerve terminal sprouting in living frog neuromuscular junctions. J Neurosci 14:796-808.

Chen L, Folsom DB, Ko CP (1991) The remodeling of synaptic extracellular matrix and its dynamic relationship with nerve terminals at living frog neuromuscular junctions. J Neurosci 11:2920-2930.

Dai ZS, Peng HB (1998) Fluorescence microscopy of calcium and synaptic vesicle dynamics during synapse formation in tissue culture. J Histochem 30:189-196.

Diaz J, Pécot-Dechavassine M (1989) Terminal nerve sprouting at the frog neuromuscular junction induced by prolonged tetrodotoxin blockade of nerve conduction. J Neurocytol 18:39-46.

Diaz J, Molgo J, Pécot-Dechavassine M (1989) Sprouting of frog motornerve terminals after long term paralysis by botulinum type A toxin. Neurosci Lett 96:127-132.

Ecker A (1889) The anatomy of the frog, pp 96, 109. Amsterdam: Asher.

Engert F, Bonhoeffer T (1999) Dendritic spine changes associated with hippocampal long-term synaptic plasticity. Nature 399:66-70.

Friedman HV, Bresler T, Garner CC, Ziv NE (2000) Assembly of new individual excitatory synapses: time course and temporal order of synaptic molecule recruitment. Neuron 27:57-69.

Georgiou J, Charlton MP (1999) Non-myelin-forming perisynaptic schwann cells express protein zero and myelin-associated glycoprotein. Glia 27:101-109.

Harris LW, Purves D (1989) Rapid remodeling of sensory endings in the corneas of living mice. J Neurosci 9:2210-2214.

Harrold J, Ritchie J, Nicholls D, Smith W, Bowman D, Pocock J (1997) The development of $\mathrm{Ca}^{2+}$ channel responses and their coupling to exocytosis in cultured cerebellar granule cells. Neuroscience 77:683-694.

Henkel AW, Lubke J, Betz WJ (1996) FM1-43 dye ultrastructural local- 
ization in and release from frog motor nerve terminals. Proc Natl Acad Sci USA 93:1918-1923.

Herrera AA, Banner LR, Nagaya N (1990) Repeated, in vivo observation of frog neuromuscular junctions: remodelling involves concurrent growth and retraction. J Neurocytol 19:85-99.

Hill RR, Robbins N (1991) Mode of enlargement of young mouse neuromuscular junctions observed repeatedly in vivo with visualisation of pre- and postsynaptic borders. J Neurocytol 20:183-194.

Ko C-P (1981) Electrophysiological and freeze-fracture studies of changes following denervation at frog neuromuscular junctions. J Physiol (Lond) 321:627-639.

Ko CP (1984) Regeneration of the active zone at the frog neuromuscular junction. J Cell Biol 98:1685-1695.

Ko CP (1985) Formation of the active zone at developing neuromuscular junctions in larval and adult bullfrogs. J Neurocytol 14:487-512.

Ko CP, Chen L (1996) Synaptic remodeling revealed by repeated in vivo observations and electron microscopy of identified frog neuromuscular junctions. J Neurosci 16:1780-1790.

Kuromi H, Kidokoro Y (1999) The optically determined size of exo/ endo cycling vesicle pool correlates with the quantal content at the neuromuscular junction of Drosophila larvae. J Neurosci 19:1557-1565.

Lichtman JW, Magrassi L, Purves D (1987) Visualization of neuromuscular junctions over periods of several months in living mice. J Neurosci $7: 1215-1222$

Lin YQ, Brain KL, Nichol KA, Morgan JJ, Bennett MR (1996) Vesicleassociated proteins and calcium in nerve terminals of chick ciliary ganglia during development of facilitation. J Physiol (Lond) 497:639-656.

Liou JC, Chen YH, Fu WM (1999) Target-dependent regulation of acetylcholine secretion at developing motorneurons in Xenopus cell cultures. J Physiol (Lond) 517:721-730.

Llinas R, Steinberg IZ, Walton K (1981) Relationship between presynaptic calcium current and postsynaptic potential in squid giant synapse. Biophys J 33:323-351.

Macleod GT, Khurana V, Gibson WG, Bennett MR (1998) Probability of quantal secretion and the mobilization of vesicles at the active zones of endplates. J Theor Biol 191:323-334.

Macleod GT, Gan JB, Bennett MR (1999) Vesicle-associated proteins and quantal release at single active zones of amphibian (Bufo marinus) motor-nerve terminals. J Neurophysiol 82:1133-1146.

Maletic-Savatic M, Malinow R, Svoboda K (1999) Rapid dendritic morphogenesis in CA1 hippocampal dendrites induced by synaptic activity. Science 283:1923-1927.

Neher E (1998) Vesicle pools and $\mathrm{Ca}^{2+}$ microdomains: new tools for understanding their roles in neurotransmitter release. Neuron 20:389-399.

Okabe S, Kim HD, Miwa A, Kuriu T, Okado H (1999) Continual remodeling of postsynaptic density and its regulation by synaptic activity. Nat Neurosci 2:804-811.

O'Malley JP, Waran MT, Balice-Gordon RJ (1999) In vivo observations of terminal Schwann cells at normal, denervated, and reinnervated mouse neuromuscular junctions. J Neurobiol 38:270-286.

Purves D, Voyvodic JT, Magrassi L, Yawo H (1987) Nerve terminal remodeling visualized in living mice by repeated examination of the same neuron. Science 238:1122-1126.

Quigley PA, Msghina M, Govind CK, Atwood HL (1999) Visible evidence for differences in synaptic effectiveness with activity-dependent vesicular uptake and release of FM1-43. J Neurophysiol 81:356-370.

Rettig J, Heinemann C, Ashery U, Sheng ZH, Yokoyama CT, Catterall WA, Neher E (1997) Alteration of $\mathrm{Ca}^{2+}$ dependence of neurotransmitter release by disruption of $\mathrm{Ca}^{2+}$ channel/syntaxin interaction. J Neurosci 17:6647-6656.

Robbins N, Polak J (1988) Filopodia, lamellipodia and retractions at mouse neuromuscular junctions. J Neurocytol 17:545-561.

Robitaille R (1998) Modulation of synaptic efficacy and synaptic depression by glial cells at the frog neuromuscular junction. Neuron 21:847-855.

Son YJ, Thompson WJ (1995) Nerve sprouting in muscle is induced and guided by processes extended by Schwann cells. Neuron 14:133-141.

Toni N, Buchs PA, Nikonenko I, Bron CR, Muller D (1999) LTP promotes formation of multiple spine synapses between a single axon terminal and a dendrite. Nature 402:421-425.

Trachtenberg JT, Thompson WJ (1997) Nerve terminal withdrawal from rat neuromuscular junctions induced by neuregulin and Schwann cells. J Neurosci 17:6243-6255.

Wernig A, Herrera AA (1986) Sprouting and remodelling at the nervemuscle junction. Prog Neurobiol 27:251-291.

Wernig A, Pécot-Dechavassine M, Stöver H (1980) Sprouting and regression of the nerve at the frog neuromuscular junction in normal conditions and after prolonged paralysis with curare. J Neurocytol 9:277-303.

Wigston DJ (1989) Remodeling of neuromuscular junctions in adult mouse soleus. J Neurosci 9:639-647.

Wu LG, Betz WJ (1999) Spatial variability in release at the frog neuromuscular junction measured with FM1-43. Can J Physiol Pharmacol 77:672-678.

Zefirov AL, Cheranov SY (1995) Changes in the topography of transmitter release in a neuromuscular synapse reflect plastic alterations occurring in active zones. Neurophysiol 27:201-207.

Zefirov AL, Benish TV, Fatkullin NF (1990) Identification of transmitter release sites in the motor nerve terminal. Neurophysiol 22:228-235.

Zoran MJ, Funte LR, Kater SB, Haydon PG (1993) Neuron-muscle contact changes presynaptic resting calcium set-point. Dev Biol 158:163-171. 